\title{
Assessing the Department of Defense's Approach to Reducing Mental Health Stigma
}

W hen facing mental health problems, many service members choose not to seek needed help because of the stigma associated with mental health disorders and treatment. Not seeking appropriate mental health care can negatively impact the quality of life and the social, emotional, and cognitive functioning of affected service members. The stigma of seeking mental health treatment in the military persists despite the efforts of both the U.S. Department of Defense (DoD) and the Veterans Health Administration to enhance mental health services. The service branches have been actively engaged in developing policies, programs, and campaigns to reduce stigma and increase service members' help-seeking behavior.

RAND sought to provide a comprehensive assessment of the effectiveness of these stigma-reduction efforts to evaluate their alignment with service members' needs and evidencebased practices and to offer recommendations on how the efforts might be improved. We started by defining what mental health stigma means in the military context, as well as assessing its prevalence in the military and the medical and societal costs. We then looked at what the scientific evidence base shows about the most-promising program and policy options for reducing stigma and evaluated how well DoD's program and policies align with that evidence base. Finally, we identified priorities for $\mathrm{DoD}$ to consider in an effort to enhance and refine its stigma-reduction efforts.

\section{Findings}

Defining mental health stigma in the military is critical to understanding how best to address the challenges it presents. Mental health stigma is a dynamic process by which a person perceives or internalizes a negative image about himself or herself or people with mental health disorders. This process happens through an interaction between a person and the key contexts in which he or she operates: public (i.e., military culture and norms), institutional (i.e., military policies and programs), social (i.e., family and friends), and individual.

Through literature review and our own analysis, we found that four immediate outcomes are empirically linked to stigma: coping mechanisms for stress, interpersonal outcomes (e.g., self-esteem), attitudes toward treatment seeking,

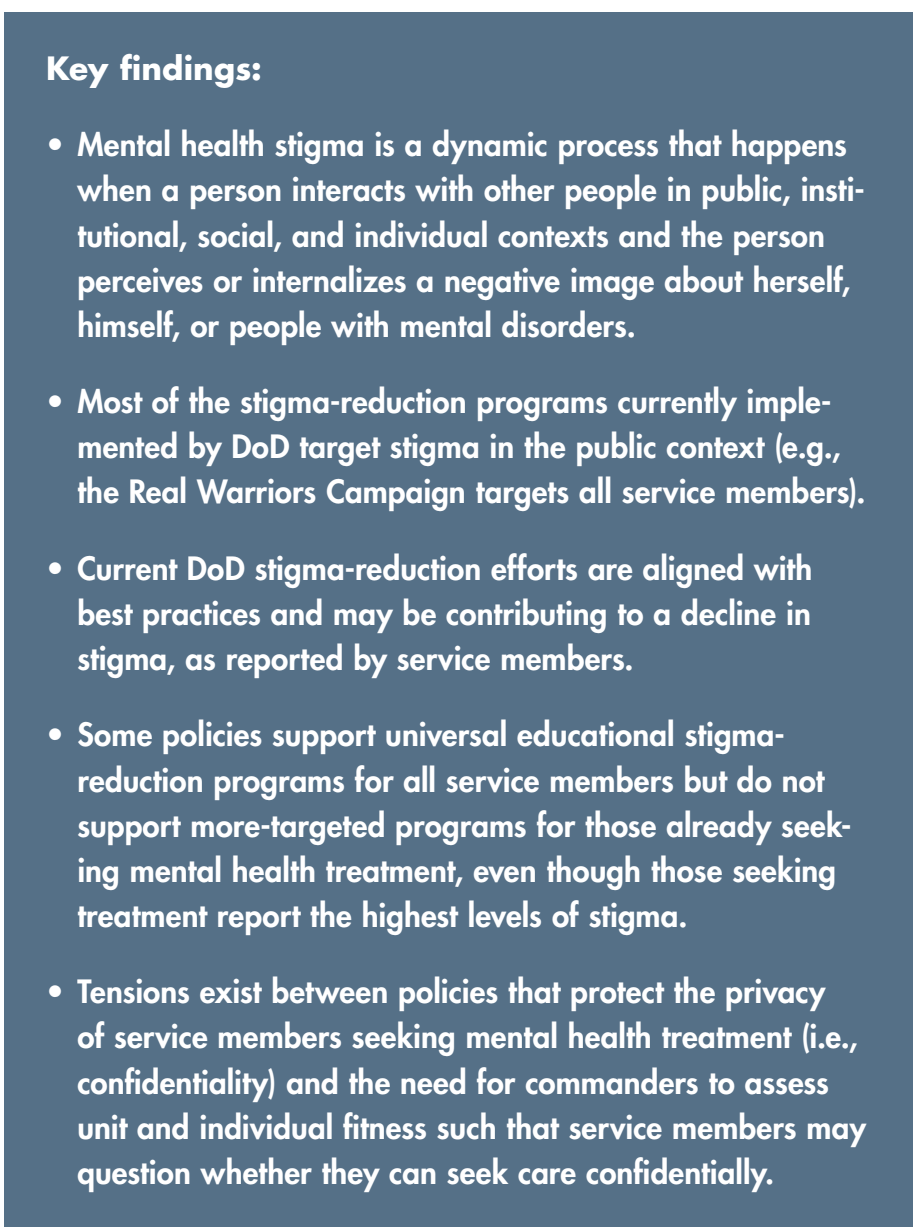

and intentions to seek treatment. Additionally, the literature has theoretically linked four long-term outcomes to stigma: well-being, quality of life, treatment initiation, and treatment success; however, these outcomes could not be empirically linked through either literature or our analysis. (See figure.)

Overall, current stigma-reduction efforts within DoD are aligned with best practices and may be contributing to a decline in self-reported stigma. For example, DoD's approach to addressing this challenge goes beyond just stigma reduction and encompasses a broader approach to minimizing barriers to mental health care. This approach is consistent with best practices and may have contributed to declines in self-reported perceptions of stigma among a subset of the 


\section{Conceptual model of stigma reduction in the military}

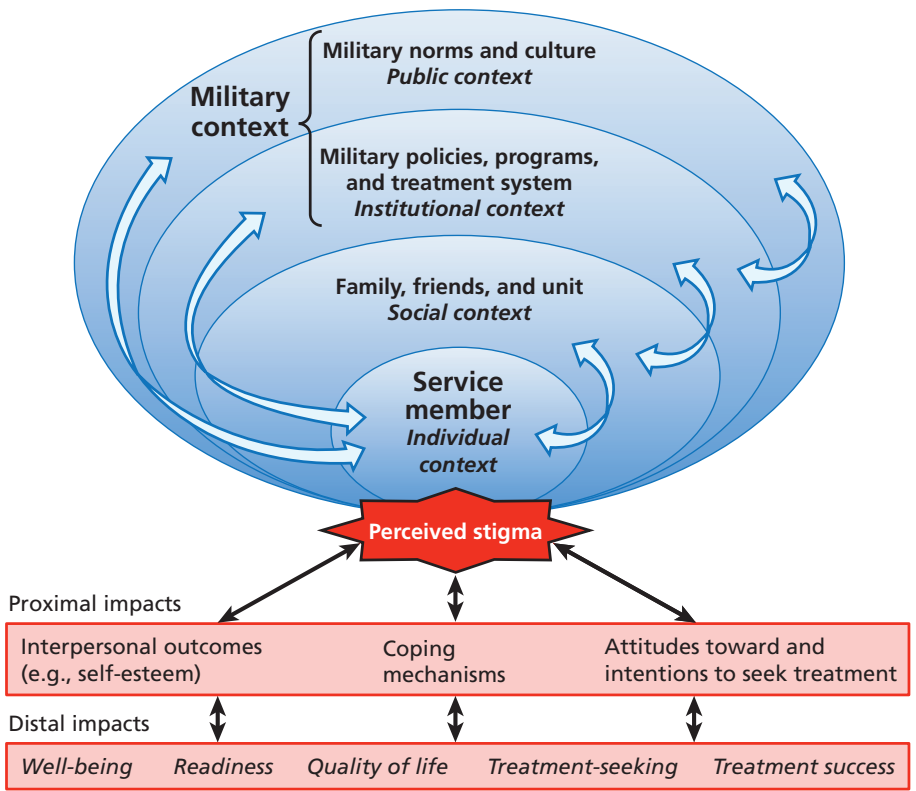

military population. Another finding of note is that most of the stigma-reduction programs currently implemented by DoD target stigma in the public context.

Our analysis also identified some challenges to DoD's efforts, including the existence of policy language barring service members with mental health disorders from career opportunities, which could create paths for discrimination, as well as tensions between policies that protect the privacy of service members seeking mental health treatment and the need for commanders to assess individual and unit fitness. Additionally, some policies support universal educational stigma-reduction programs but do not support more-targeted programs for those who are already seeking mental health treatment.

\section{Recommendations}

To improve the effectiveness of DoD's stigma-reduction efforts, RAND makes the following recommendations:

- Convene a task force to explore the tensions between a service member's privacy and a commander's need to know a service member's mental health status and treatment history.

- Explore interventions that directly increase treatmentseeking beyond just stigma reduction.

- Put evidence-based approaches in place that empower service members who have mental health concerns to support their peers.

- Develop new or tailor existing intervention-delivery mechanisms to minimize operational barriers for service members seeking treatment.

- Embed stigma-reduction interventions in clinical treatment.

- Implement and evaluate stigma-reduction programs targeting service members who have not yet developed symptoms of mental illness.

- Review and modify policies that contribute to mental health stigma, such as those that can limit job opportunities and that contain stigmatizing language.

- Improve research and evaluation related to stigma reduction by assessing the modifications made to existing programs that aim to address stigma and other barriers to care.

- Examine the dynamic nature of stigma and how it interacts with internal and external conditions over time.

- Improve measures of prevalence to improve tracking of stigma and other barriers to care.

- Review classified departmental and service-specific policies to determine potential implications for mental health stigma and discrimination.

This research brief describes work done in the RAND National Defense Research Institute and documented in Mental Health Stigma in the Military, by Joie D. Acosta, Amariah Becker, Jennifer L. Cerully, Michael P. Fisher, Laurie T. Martin, Raffaele Vardavas, Mary Ellen Slaughter, and Terry L. Schell, RR-426-OSD (available at www.rand.org/t/RR426), 2014. To view this brief online, visit www.rand.org/t/RB9881. The RAND Corporation is a research organization that develops solutions to public policy challenges to help make communities throughout the world safer and more secure, healthier and more prosperous. RAND is nonprofit, nonpartisan, and committed to the public interest. RAND's publications do not necessarily reflect the opinions of its research clients and sponsors. RAND ${ }^{\circledast}$ is a registered trademark. ๑ RAND 2016

Limited Print and Electronic Distribution Rights: This document and trademark(s) contained herein are protected by law. This representation of RAND intellectual property is provided for noncommercial use only. Unauthorized posting of this publication online is prohibited. Permission is given to duplicate this document for personal use only, as long as it is unaltered and complete. Permission is required from RAND to reproduce, or reuse in another form, any of our research documents for commercial use. For information on reprint and linking permissions, please visit www.rand.org/pubs/permissions.html. 